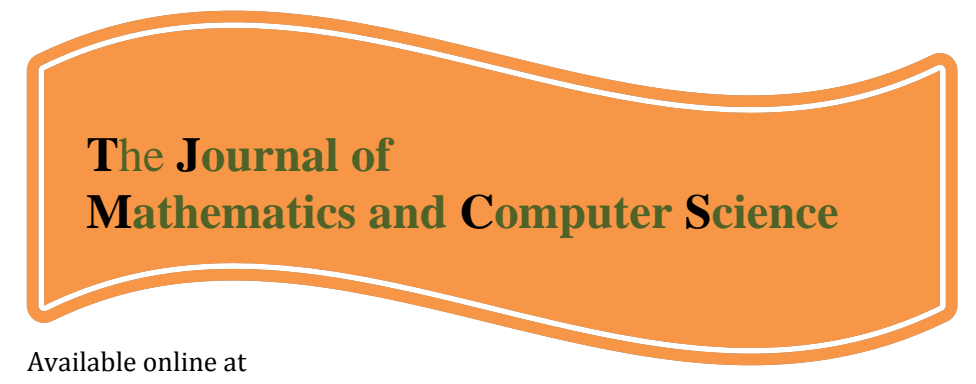

http://www.TJMCS.com

The Journal of Mathematics and Computer Science Vol .2 No.2 (2011) 222-232

\title{
A Novel Fuzzy Expert System Using Image Processing for Sale Car Shape with Online Membership Function
}

\author{
Seyyed Mohammad Reza Farshchi1, ${ }^{*}$, Mahdi Yaghoobi² \\ Department of Artificial Intelligence, Islamic Azad University, Mashhad Branch, \\ Shiveex@gmail.com \\ Department of Artificial Intelligence, Islamic Azad University, Mashhad Branch, \\ Yaghoobi@mshdiau.ac.ir
}

Received: August 2010, Revised: November 2010

Online Publication: January 2011

\begin{abstract}
In This paper we proposed a novel fuzzy retrieval system for purchasing cars employing image processing. The car shape is an important factor when selecting a car type. This system aims to support persons who are not good with cars. When we try to purchase a car, they can use this system easily as if they ask casually someone else who knows more about car. Unspecific conditions are expressed by the fuzzy set, and the level matching conditions are expressed by the grade values. To use this more practically, a GUI form with selection menus is developed. This system is designed to change membership function automatically for improving usability of this system. In addition, calculating curvature by the car shape using the image processing, and adding items for selecting a car shape from roundness and sharpness. Furthermore, the effectiveness of applying fuzzy logic to express man's subjectivity when selecting car type.
\end{abstract}

Keywords: Fuzzy logic, Online Membership, Fair Car, Fuzzy Retrieval System.

\footnotetext{
$1,{ }^{*}$ Associated Professor in Imaging Science and Engineering Lab., Sadjad University of Mashhad.

${ }^{2}$ Associated Professor in Artificial Intelligence, Islamic Azad University, Mashhad Branch.
} 


\section{Introduction}

As car-oriented society has grown these days, the number of cars owned by one household is increasing. It is even no longer one car per household, its one car per person. Car makers provide variety of unique cars suit for individuals It is fun for people to choose car type, especially for those who have special interest in cars. However, for opposites it's relatively difficult to choose cars that suit them most Generally, when people who don't have special interest in cars search for car information over internet and if they don' know or remember appropriate keywords to be input, they may not get satisfied search results. This study therefore uses

fuzzy theory [1][2][3][4], which manages daily and sensuous words, aiming at developing a practical car retrieval system and at proving their effectiveness. A GUI form as a supportive system for purchasing cars is developed [5]. In this form, unspecific words used in daily conversations can be used for searching (Such words are like "relatively large in size", "relatively high power", etc.), and there is no need of special knowledge of cars. In addition, an improved system which enables to set importance of each item is adopted for a usability test, and it shows good result [10]. After the test, some testers requested more selection items based on the car appearance, so car shapes such as "roundness" and "sharpness" are newly added.

\section{Image Processing and Curvature for Car Shape}

Curvature, the degree of a curve, is adopted as an indicator for the car shape such as roundness and sharpness. It is calculated by the inverse number of a radius of curvature. A circle of curvature at point $\mathrm{A}$ on the successive curved line is defined as a circle which passes through the point $A$ and neighboring two points. As for a discrete system, the circle of curvature at $A\left(x_{i}, y_{i}\right)$ is calculated as a circle passes through $A\left(x_{i}, y_{i}\right)$, neighboring points $B\left(x_{i-1}, y_{i-1}\right)$ and $C\left(x_{i+1}, y_{i+1}\right)$. Assuming that the curvature at $\mathrm{A}$ is $k_{i}$ and the radius is $r_{i}$, the result is obtained by the formula of the sine theorem and the cross product as below:

$$
k_{i}=\frac{1}{r_{i}}=\frac{2 \sin A}{B C}=\frac{2 \cdot(\overline{A B} \times \overline{A C})}{B C \cdot A B \cdot A C}
$$

Now, the cross product and the lengths between each point are expressed in coordinates as follows:

$$
\begin{aligned}
& \overline{A B} \times \overline{A C}=\left(x_{i-1}-x_{i}\right)\left(y_{i+1}-y_{i}\right)-\left(x_{i+1}-x_{i}\right)\left(y_{i-1}-y_{i}\right) \\
& B C=\sqrt{\left(x_{i-1}-x_{i+1}\right)^{2}+\left(y_{i-1}-y_{i+1}\right)^{2}} \\
& A B=\sqrt{\left(x_{i-1}-x_{i}\right)^{2}+\left(y_{i-1}-y_{i}\right)^{2}} \\
& A C=\sqrt{\left(x_{i+1}-x_{i}\right)^{2}+\left(y_{i+1}-y_{i}\right)^{2}}
\end{aligned}
$$

\subsection{Calculation of an Indicator for Car Shape}

To obtain a comprehensive indicator for the car shape, $\mathrm{K}$, outline of the car is extracted from the car image, then the curvatures of every dots on coordinate is calculated by those average. 


$$
k=\frac{x_{\max }-x_{\min }}{l} \cdot \frac{1}{n} \sum_{i=1}^{n} k_{i}
$$

Where, $x_{\max }$ and $x_{\min }$ indicate the maximum value and the minimum value on the coordinate of the image, respectively. $\mathrm{L}$ is the car length $(\mathrm{m})$ and $\mathrm{n}$ is the number of data. The image is supposed to show a side view of the car, and its size and actual car size are corrected.

\section{Expert Systems Component}

In this section we will explore the emulation of human thought, capable of dealing with uncertainties, ambiguities, and contradictions. We agree with Anderson [5] that much human thought can be expressed in rules [6]. To handle uncertainties, ambiguities, and contradictions, we will use fuzzy systems techniques, implemented by a fuzzy expert system.

Expert systems are computer programs, designed to make available some of the skills of an expert to non experts. Since such programs attempt to emulate in some way an expert's thinking patterns, it is natural that the first work here was done in Artificial Intelligence (AI) circles. Among the first expert systems were the 1965 Dendral programs [5], which determined molecular structure from mass spectrometer data; R1 [6] used to configure computer systems; and MYCIN [7] for medical diagnosis. Since the middle 1960s there have been many expert systems created for fields ranging from space shuttle operations through intensive-care-unit patient alarm systems to financial decision making.

There is a variety of ways in which the problem of creating computer programs to act like an expert has been approached; a valuable reference is Jackson [8]. One of the earliest methods employs rule-based systems, which use "If ... Then..." rules to represent the expert's reasoning process (if the data meet certain specified conditions, then take appropriate actions). Other approaches include semantic or associative nets [2], frames [3] and neural nets [4], currently very popular in a wide variety of fields of these, clearly dominant are the complementary rule-based systems and neural net approaches.

Neural nets do not require that the thinking patterns of an expert be explicitly specified. Instead, two sets of data are required from the real world. These data include all the inputs to the system, and the correct outputs corresponding to these input values. The first data set or training set is used to train the neural network so that, as nearly as possible, the correct outputs are produced for each set of input values. The second data set or validation set is used after the neural net has been trained to make sure that correct answers are produced on different input data. An advantage of neural nets is that it is not necessary to extract the thinking patterns of the expert and to render these explicit. Disadvantages are that a substantial training set is required, and that while the neural net may produce reasonably correct answers, in general, we have little or no idea how it does this. Considerable work has been done on extracting rules from a trained neural net, but this work is not yet advanced to a very satisfactory state.

Rule-based systems require that the expert's knowledge and thinking patterns be explicitly specified. Usually two persons (or groups) develop a system. These are the domain expert, who knows how to solve the problem at hand but who is seldom acquainted with computer programming; and the knowledge engineer, who is thoroughly familiar with the computer technology involved and expert systems but who has little or no knowledge of the problem at hand. Obtaining this knowledge and writing proper rules is called the knowledge acquisition phase [7].

After the system has been written, it must be tuned for accuracy using a Tuning data set similar to the training set of a neural net, but usually much smaller. After tuning, a rule based system must be 
validated in the same way as a neural net. Rule-based systems have two advantages. A large training set is usually not required, and since the expert's thinking is explicitly spelled out we now know how he thinks about the problem. They have the disadvantage that the knowledge acquisition phase may be difficult. A great advantage of fuzzy expert systems is that most rules can be written in language that the expert can directly understand, rather than in computer jargon; communication between domain expert and knowledge engineer is greatly eased.

Another advantage of rule-based expert systems is the potential ability of rule based expert systems to learn by creation of new rules and addition of new data to the expert knowledge data base. Probably the first example of a rule-based expert system to rival human experts was DENDRAL, which deduced the molecular structure of organic compounds from knowledge about fragments into which the compound had been [4]. One set of DENDRAL's programs worked directly with the data to produce candidate structures. An additional program, Meta-DENDRAL, worked directly with the DENDRAL rules to improve them and discover new rules [9], thus discovering new concepts about the data. Meta-DENDRAL was not itself written as a rule-based expert system, but the ability of a rule to generate new rules and new expert factual knowledge opens the possibility for writing expert systems that can create new rules and store new expert factual knowledge. This exciting possibility has not as yet been well explored, perhaps due to the common (and, we think, quite incorrect) assumption among conventional AI practitioners that expert systems are no longer to be considered as artificial intelligence.

\subsection{Fuzzy Information Retrieval System}

\subsubsection{GUI form and the important degrees}

Figure 1 shows the proposed GUI form. We employ slide objects as the importance of each item. Users can select items according to their special interests or importance Details of this form are as follows:

I. Elements of price is the crisp set, which range from 1 million yen to 10 million dollar at interval of 0.5 million dollar.

II. Each item is the fuzzy set. Five membership functions of the triangle type are adopted as stated in the next section.

(i) The car size which values are length times width.

(ii) Engine displacement which shows measurement in liters.

(iii) Maximum power which shows a measurement in kilowatts.

III. Shape is the fuzzy set. It is an item relating the car shape, which can be selected from Roundness and Sharpness.

IV. Each item is the crisp set.

(i) Type of vehicle: 2-Box car, sedan, wagon, etc.

(ii) Color: White, Silver, Black etc.

(iii) Number of Seats: An item especially for large families. 
V. Check box for the following particular uses. These items are the fuzzy sets as stated in the next section

(i) Low mileage: For emission certification and fuel economy, we adopt 10-15 mode cycle which is currently used in Japan. The 10 mode cycle simulates urban driving conditions, and the 15 mode cycle simulates suburb or highway driving conditions.

(ii) Good visibility: The height of cars.

(iii) Spacious inner volume: The cars with high ratio of the inner volume out of the whole volume.

VI. Check box for the following particular uses. These items are the crisp sets.

(i) Slide door: The car equipped with the slide doors.

(ii) Smart key system. The car equipped with the key less entry system.

(iii) 4WD: The four-wheel drive system is included in the standard specification.

(iv) Protection of the environment: Eco-friendly car which can reduce hazardous gas emission by more than 50 .

VII. Slider objects for the important degrees: The value of slider multiplied by the fuzzy or the crisp grades of each item.

VIII. Car search result. Car types and model names at all grades are listed in order of highest match rate on top.

IX. Total values are listed as fitting rate.

X. Buttons which link to the official web sites.

XI. Images of the top 3 are shown here.

\subsubsection{Membership functions}

Two membership functions are applied to the car shape. Five membership functions are applied to following items each; the car size, the engine displacement and the maximum power. Furthermore one membership function is applied to following items each; mileage, height and ratio of the inner volume (inner volume / whole volume). In general, as membership functions are identified subjectively, we adopt a theory which is based on statistics for the boundary of fuzzy area. We adopt the two standard deviations for the boundaries of fuzzy area, $A$ and $B(A<B)$ are:

$$
A=\mu-2 \sigma
$$




$$
B=\mu+2 \sigma
$$

Where $\mu$ and $\sigma$ are average and standard deviations of a data $x_{i}=(i=1,2, \ldots, n)$, respectively. These are shown as follows:

$$
\begin{aligned}
& \mu=\frac{1}{n} \sum_{i=1}^{n} x_{i} \\
& \sigma=\sqrt{\frac{1}{n} \sum_{i=1}^{n}\left(x_{i}-\mu\right)^{2}}
\end{aligned}
$$

Figure 2 shows the membership functions for the car shape. Matching level between the selected element and the car data by using this function.

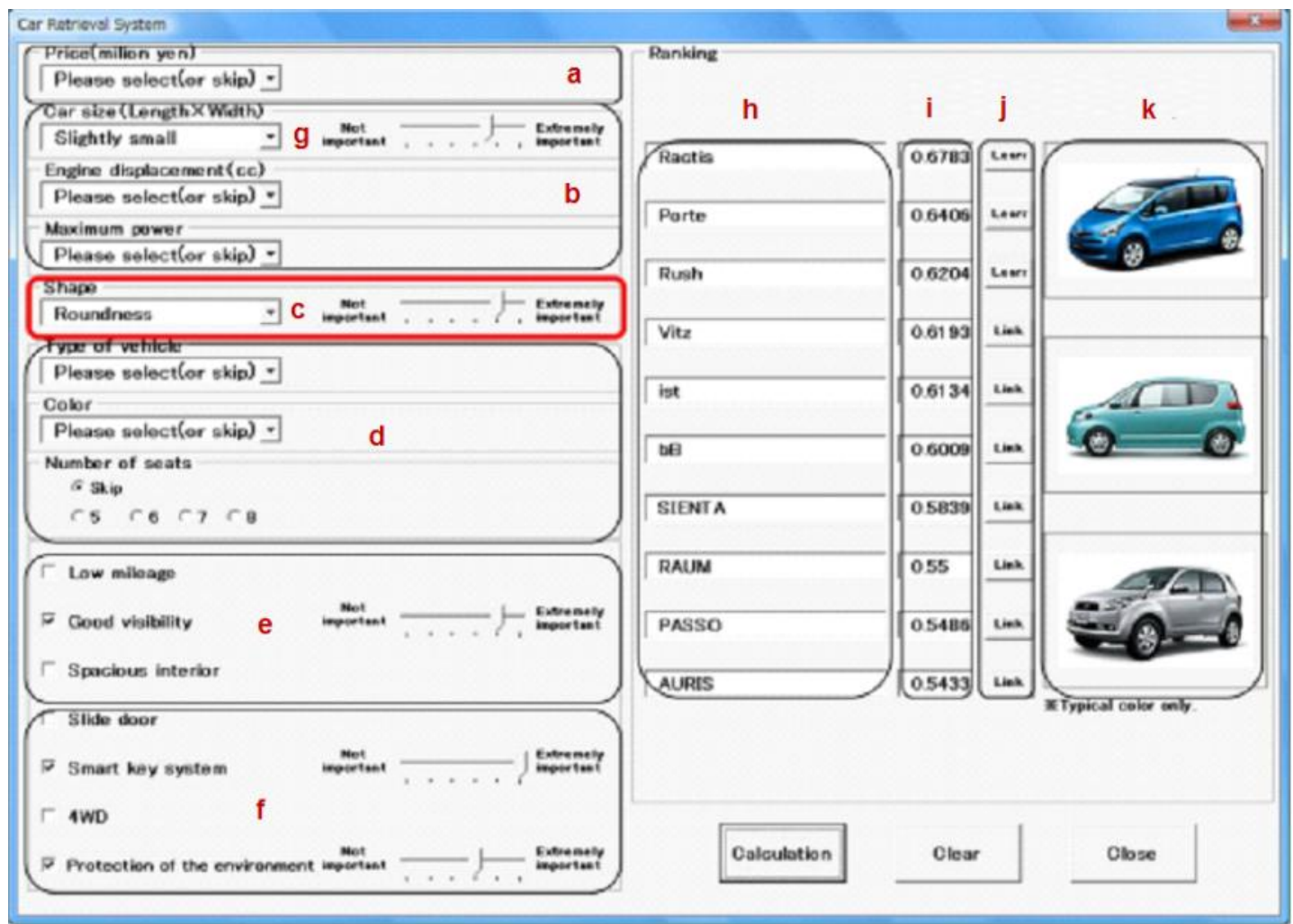

Figure 1. Proposed GUI form for Car Retrieval System

\section{Car data}

The car maker used in the car retrieval system is selected to IRICO, which has a largest share in the industry of Iran, in order to improve the accuracy of the system. Selectable models are 49 types of current models excluding used ones (excluding the full size models from the total 52 types (as of May 2009)). To reflect user feeling, we adopt the sales volumes in Mashhad from 2000 to 2009. Figure 3 shows the histograms for the car shape. Here, $\mu$ is average deviations, $\sigma$ is standard deviations of the data for the car shape. 


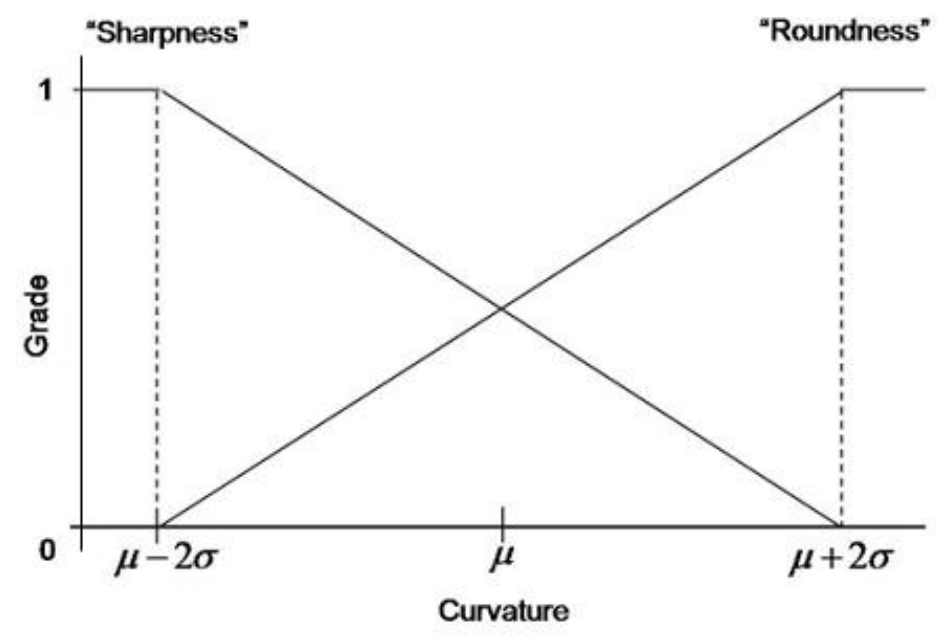

Figure 2. Membership functions for the car shape

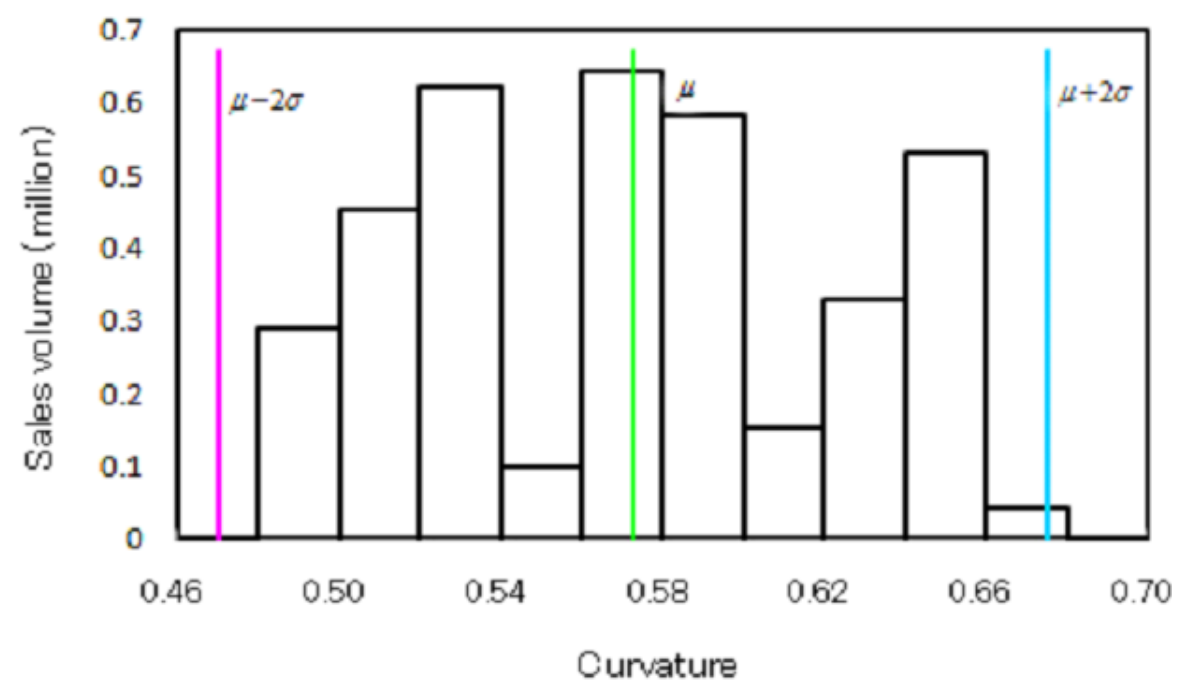

Figure 1. Histogram for the car shape

\section{Processing Flow and Calculations}

Here we describe details of this study for how to calculate the grade value for the membership function actually used, and explains calculation of fitting rate with the important degrees too. The processing flow of the car retrieval system described as followed: Conclusion may review the main points of the paper, do not replicate the abstract as the conclusion. It might elaborate on the importance of the work or suggest applications and extensions.

(i) Selecting elements from each item. Users can skip items they don't know well or are not interested etc.

(ii) The grades of the crisp sets and the fuzzy sets are calculated as following. 
Crisp set:

$$
a_{k}=\left\{\begin{array}{l}
1 \\
0
\end{array}\right.
$$

Fuzzy set:

$$
a_{k}=f_{t}(x)
$$

Where $f_{t}(x)$ is the membership function shown in Figure 2, and $k$ is the number of items of crisp sets and fuzzy sets. $a_{k}$ Indicates the matching level of selected element and car data.

(iii) Calculations: The values of slider object shows as the important degrees for each element. Fitting rate for all cars are calculated as follows:

$$
P=\frac{1}{m} \sum_{k=1}^{m} w_{k} \cdot a_{k}
$$

Where, $m$ is the number of item which selected in proposed fuzzy information retrieval system. In addition, $\mathrm{P}$ has a range of 0 to 1.

(iv) The car models are shown in order, the highest fitting rate on top.

\section{Experimental Result}

Operation of the item of the newly-added image processing is checked here. On item "Shape", Figure 4 shows the result of selecting "Roundness", and Figure 5 shows the result of "Sharpness". Table 1 shows the top 5 resulted from each shape. In order to output the result as it is, all other items are omitted and the slider is adjusted to the maximum. As shown in the images in Figure 4 and 5, the size of the cars resulted from "Roundness" is relatively small, and those from "Sharpness" is relatively large. It is because there is a correlation between people who prefer small cars and those prefer round cards. The same applies to the large cars. In addition, larger ratio of straight line is expected for the large cars due to legal car size.

Table 1. Result of selecting each element of "shape"

\begin{tabular}{lllll}
\hline Rank & Car Shape "Roundness" & Fuzzy Grades & Car Shape "Sharpness" & Fuzzy Grade \\
\hline 1 & AURIS & 0.85 & TOYOTA & 0.94 \\
2 & Ractis & 0.64 & PARS & 0.91 \\
3 & Vitz & 0.97 & 206 & 0.90 \\
4 & PASSO & 0.88 & 405 & 0.86 \\
5 & Ist & 0.76 & PRADO & 0.84 \\
\hline
\end{tabular}




\section{Conclusion}

In this paper, we proposed the fuzzy information retrieval system employing the image processing for the car shape. The car shape is an important factor when selecting a car type. By employing this factor as a selection item, usability of this fuzzy retrieval system is improved. Since it is a simple selection from "Roundness" and "Sharpness", even people who don't know well about cars can search a car with this supportive system casually. Unspecific conditions are expressed by the fuzzy set, and the level matching the conditions are expressed by the grade values.

Big reason for adopting the fuzzy logic in this study is to express man's subjectivity and fuzziness, but the logic does not always fit to everyone because each person has different subjectivity. Challenges for the future are to have questionnaires over the internet, and to adopt a system allowing changing membership function automatically to make the fuzzy set closer to the sense of users. In addition, an automatic data updating system which cruises around the web is considered to be established, allowing reflecting the information from many car makers on car types, used cars, and even grades of each car type.

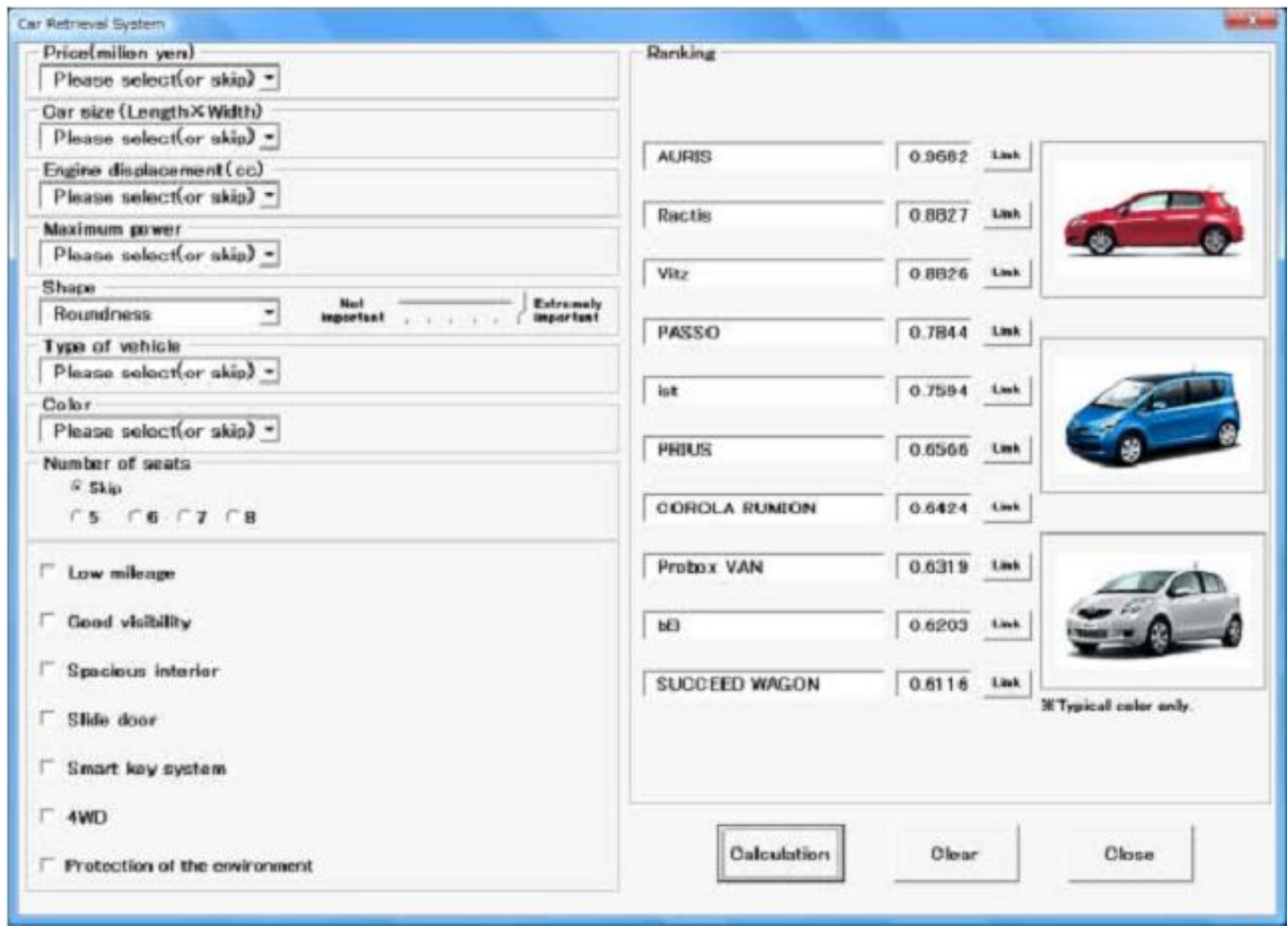

Figure 4. Result of selecting "Roundness" 
Seyyed Mohammad Reza Farshchi, Mahdi Yaghoobi/ TJMCS Vol .2 No.2 (2011) 222-232

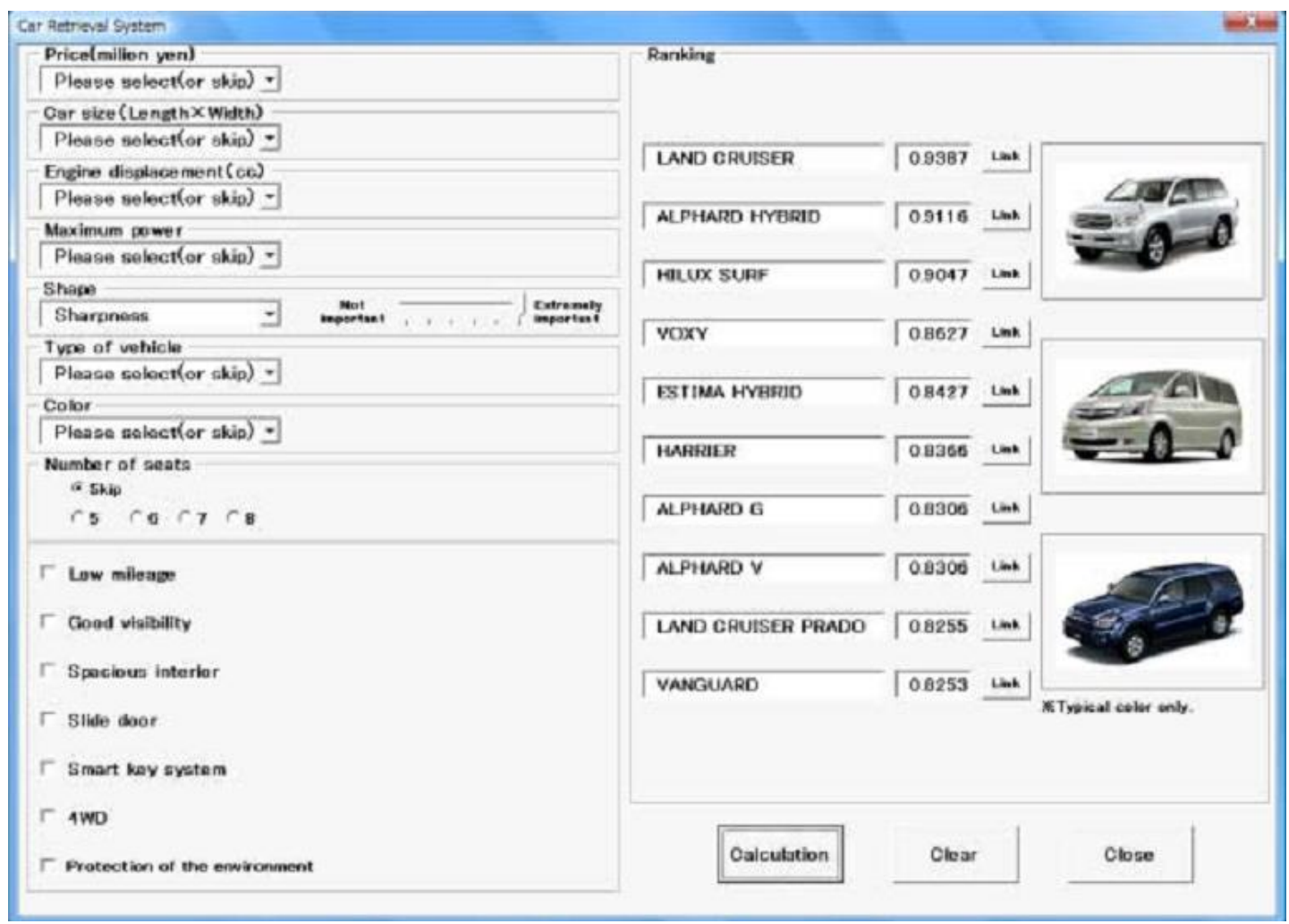

Figure 5. Result of selecting "Sharpness"

\section{References}

[1] Dubois, D., and Henri, M., "Fuzzy Sets and Systems: Theory and Applications", Academic Press, 1980.

[2] Schmucker, K.J., "Fuzzy Sets, Natural Language Computations, and Risk Analysis", Computer Science Press, 1984.

[3] Klir, G.L., and Bo Yuan, "Fuzzy Sets and Fuzzy Logic Theory and Applications", Prentice-Hall International, 1995.

[4] Zadeh, L.A., "Fuzzy sets", Information and Control, Vol. 8, No. 3, pp. 338-358, 1968.

[5] Samatsu, T., Tachikawa, K., and Shi, Y., "GUI form for car retrieval systems using fuzzy theory", ICIC Express Letters, Vol. 2, No. 3, pp. 245-249, 2008.

[6] Samatsu, T., Tachikawa, K., and Shi, Y,. "Usability improvement for a car retrieval system employing the important degrees of fuzzy grades", International Journal of Innovative Computing, Vol. 14, No. 3, 2007.

[7] Rosenfeld, A., and Johnston, E., "Angle detection on digital curves", IEEE Computer Society, Vol. C-22, Issue 9, pp. 875-878, 1973. 
[8] Farin, G., and Sapidis, N., "Curvature and the fairness of curves and surfaces", IEEE Computer Society, Vol. 9, Issue 2, pp.52-57, 1989.

[9] Chang, F.C., and Hang, H.M., "A relevance feedback image retrieval scheme using multi-instance and pseudo image concepts", IEICE Trans. on Information and Systems, Vol. E89-D, No. 5, pp. 1720-1731, 2006.

[10] Jang, J.S., "ANFIS: adaptive network based fuzzy inference system", IEEE Transaction on System and Management, Vol. 23, pp. 665-685, 1993. 